\title{
COMPACTIFICATIONS OF TOTALLY BOUNDED QUASI-UNIFORM SPACES
}

\author{
by P. FLETCHER and W. F. LINDGREN
}

(Received 10 October, 1984)

1. Introduction. The notation and terminology of this paper coincide with that of reference [4], except that here the term, compactification, refers to a $T_{1}$-space. It is known that a completely regular totally bounded Hausdorff quasi-uniform space $(X, \mathscr{V})$ has a Hausdorff compactification if and only if $\mathscr{V}$ contains a uniformity compatible with $\mathscr{T}(\mathscr{V})$ [4, Theorem 3.47]. The use of regular filters by E. M. Alfsen and J. E. Fenstad [1] and $O$. Njåstad [5], suggests a construction of a compactification, which differs markedly from the construction obtained in [4]. We use this construction to show that a totally bounded $T_{1}$ quasi-uniform space has a compactification if and only if it is point symmetric. While it is pleasant to have a characterization that obtains for all $T_{1}$-spaces, the present construction has several further attributes. Unlike the compactification obtained in [4], the compactification given here preserves both total boundedness and uniform weight, and coincides with the uniform completion when the quasi-uniformity under consideration is a uniformity. Moreover, any quasi-uniformly continuous map from the underlying quasiuniform space of the compactification onto any totally bounded compact $T_{1}$-space has a quasi-uniformly continuous extension to the compactification. If $u$ is the Pervin quasi-uniformity of a $T_{1}$-space $X$, the compactification $(\breve{X}, \mathscr{T}(\breve{\mathscr{U}}))$ we obtain is the Wallman compactification of $(X, \mathscr{T}(U))$. It follows that our construction need not provide a Hausdorff compactification, even when such a compactification exists; but we obtain a sufficient condition in order that our compactification be a Hausdorff space and note that this condition is satisfied by all uniform spaces and all normal equinormal quasi-uniform spaces. Finally, we note that our construction is reminiscent of the completion obtained by $\dot{A}$. Császár for an arbitrary quasi-uniform space [2, Section 3]; in particular our Theorem 3.7 is comparable with the result of [2, Theorem 3.5].

2. Preliminary results. For the sake of completeness, we begin by citing some definitions given in reference [4]. A quasi-uniform space $(X, \mathscr{U})$ is point symmetric provided that for each $U \in \mathscr{U}$ and $x \in X$ there is a symmetric $V \in \mathscr{U}$ such that $V(x) \subset U(x)$. It is useful to observe that $U$ is point symmetric if and only if $\mathscr{T}(U) \subset \mathscr{T}\left(U^{-1}\right)$. Evidently, if $U$ contains a uniformity compatible with $\mathscr{T}(\mathscr{U})$, then $U$ is point symmetric; the converse is false even for completely regular quasi-uniform spaces. Every compact $T_{1}$-space is point symmetric; and, since every quasi-uniform subspace of a point-symmetric quasi-uniform space is point symmetric, point symmetry is a necessary condition for a quasi-uniform space to have a compactification.

If $(X, U)$ is a quasi-uniform space, $U^{*}$ denotes the coarsest uniformity that contains $\mathcal{U}$ and, for each $x \in X, \eta_{x}^{*}$ denotes the $\mathscr{T}\left(\mathcal{U}^{*}\right)$-neighborhood filter of $x$. A filter $\mathscr{F}$ on a quasi-uniform space $(X, \mathscr{U})$ is a Cauchy filter provided that for each $U \in \mathscr{U}$ there is an

Glasgow Math. J. 28 (1986) 31-36. 
$x \in X$ such that $U(x) \in \mathscr{F}$. A quasi-uniform space $(X, \mathscr{U})$ is totally bounded provided that for each $U \in \mathscr{U}$ there is a finite cover $\mathscr{C}$ of $X$ so that $C \times C \subset U$ for each $C \in \mathscr{C}$. Equivalently, $(X, \mathcal{U})$ is totally bounded provided that every ultrafilter over $X$ is a $U^{*}$-Cauchy filter. If $A$ and $B$ are subsets of a set $X, T(A, B)$ denotes $X \times X-A \times B$. If $\mathcal{U}$ is a totally bounded quasi-uniformity, $\mathscr{P}=\{T(A, B)$ : for some $U \in \mathscr{U}, A \times B \cap U=\varnothing\}$ is a subbase for $U$. Each $U^{*}$-Cauchy filter $\mathscr{F}$ contains exactly one minimal $\mathcal{U}^{*}$-Cauchy filter, namely the filter that has as a base $\left\{U(F): U\right.$ is a symmetric member of $\mathscr{U}^{*}$ and $\left.F \in \mathscr{F}^{*}\right\}$.

Let $(X, U)$ be a quasi-uniform space. Then $\tilde{X}$ denotes the set of all minimal $U^{*}$-Cauchy filters on $X$, for each $U \in \mathscr{U}, \tilde{U}=\{(\mathscr{F}, \mathscr{G}) \in X \times X$ : there is an $F \in \mathscr{F}$ and a $G \in \mathscr{G}$ so that $F \times G \subset U\}$ and $\tilde{U}$ denotes the quasi-uniformity on $\bar{X}$ for which $\{\tilde{U}: U \in \mathcal{U}\}$ is a base. The pair $(\tilde{X}, \bar{U})$ is called the bicompletion of $(X, \mathscr{U})$. Since $(\tilde{U})^{*}=\left(U^{*}\right)^{\sim}$, we always write $\tilde{U}^{*}$ to denote this uniformity. It is a complete uniformity, and $(X, \mathcal{U})$ is quasi-unimorphic to a $\mathscr{T}\left(\tilde{U}^{*}\right)$-dense subspace of $(\tilde{X}, \tilde{\mathscr{U}})$.

In the study of quasi-uniform spaces, the bicompletion of a quasi-uniform space is the natural analogue of the completion of a uniform space; and, since the bicompletion $(\tilde{X}, \tilde{U})$ of a quasi-uniform space $(X, \mathcal{U})$ is compact if the quasi-uniform space is totally bounded, the bicompletion appears to provide the natural compactification of a totally bounded quasi-uniform space. Our first result rules out this red herring.

Proposition 2.1. Let $(X, \mathcal{U})$ be a totally bounded $T_{1}$ quasi-uniform space. Then $\mathscr{T}(\tilde{U})$ is a $T_{1}$ topology if and only if $U$ is a uniformity.

Proof. If $u$ is a uniformity, $\tilde{U}$ is the usual completion, which is well known to be a Hausdorff uniformity.

Now suppose that $\mathscr{T}(\tilde{U})$ is a $T_{1}$ topology. Both $\mathscr{T}(\overline{\mathscr{U}})$ and $\mathscr{T}\left(\tilde{U}^{-1}\right)$ are coarser than $\mathscr{T}\left(\bar{U}^{*}\right)$, which is compact. Thus $\tilde{U}$ and $\tilde{U}^{-1}$ are point symmetric and $\mathscr{T}(\tilde{U})=\mathscr{T}\left(\tilde{U}^{-1}\right)=$ $\mathscr{T}\left(\tilde{U}^{*}\right)$. Since $\tilde{U}$ is a $T_{1}$ quasi-uniformity, $\cap \tilde{U}=\Delta_{\bar{X}}$ and it follows that $\tilde{U}$ consists of all the $T(\tilde{U}) \times T(\tilde{U})$-neighborhoods of $\Delta_{\bar{X}}[4$, Theorem 1.20]. Evidently $\tilde{U}$ and hence $U$ is a uniformity.

A filter $\mathscr{F}$ on a quasi-uniform space $(X, \mathcal{U})$ is a regular filter provided that for each $F \in \mathscr{F}$ there exists an $E \in \mathscr{F}$ and a $U \in \mathscr{U}$ such that $U^{-1}(E) \subset F$. Note that in case $(X, \mathcal{U})$ is a uniform space the definition of a regular filter given here coincides with the definition of a regular filter given by Alfsen and Fenstad [1]. For any filter $\mathscr{F}$ on $(X, \mathcal{U}), \mathscr{F}^{r}$ denotes the filter for which $\left\{V^{-1}(F): V \in \mathscr{U}, F \in \mathscr{F}\right\}$ is a base. We omit the proof of the following proposition, since comparable results are obtained in reference [1].

Proposition 2.2. Let $\mathscr{F}$ be a filter on a quasi-uniform space $(X, \mathcal{U})$.

(a) F्F and $\mathscr{F} r$ have the same set of cluster points.

(b) Every regular filter is contained in a maximal regular filter.

(c) A regular filter $\mathscr{F}$ is a maximal regular filter if and only if either $X-A$ or $B$ belongs to $\mathscr{F}_{\mathcal{F}}$ whenever $U^{-1}(A) \subset B$.

3. Construction of a compactification. The first result of this section demonstrates the importance of total boundedness in the forthcoming construction. 
Lemma 3.1. Let $(X, \mathcal{U})$ be a quasi-uniform space. Every regular $\mathcal{U}^{*}$-Cauchy filter is a maximal regular filter, and if $(X, \mathcal{U})$ is totally bounded every maximal regular filter is a $u^{*}$-Cauchy filter.

Proof. Suppose that $\mathscr{F}$ is a regular $\mathcal{U}^{*}$-Cauchy filter on $X$, let $A$ and $B$ be subsets of $X$ and let $U$ be an entourage in $U$ such that $U^{-1}(A) \subset B$. Let $F \in \mathscr{F}$ such that $F \times F \subset U$. If $F \cap A \neq \varnothing$, then $F \subset U^{-1}(A) \subset B$ so that $B \in \mathscr{F}$. If $F \cap A=\varnothing$, then $F \subset X-A$ and $X-A \in \mathscr{F}$. It follows from Proposition 2.2(c) that $\mathscr{F}$ is a maximal regular filter.

Now suppose that $U$ is totally bounded and that $\mathscr{F}$ is a maximal regular filter. Then $\mathscr{S}=\{T(A, B)$ : for some $U \in \mathscr{U}, A \times B \cap U=\varnothing\}$ is a subbase for $\mathcal{~} U$. Let $T(A, B) \in \mathscr{P}$. Then $T(B, A)(B) \subset X-A$ so that either $X-A$ or $X-B$ belongs to $\mathscr{F}$. Since $(X-A) \times$ $(X-A) \cup(X-B) \times(X-B) \subset T(A, B)$, we have shown that $\mathscr{F}$ is a $U^{*}$-Cauchy filter.

Proposition 3.2. Let $(X, U)$ be a totally bounded quasi-uniform space and let $\mathscr{F}$ be a maximal regular filter on $X$. Then for each $U \in \mathcal{U}$ and $F \in \mathscr{F}$, there exists $a x \in F$ such that $U(x) \cap U^{-1}(x) \in \mathscr{F}$.

Proof. Let $U \in \mathscr{U}$ and $F \in \mathscr{F}$. By the preceding lemma, $\mathscr{F}$ is a $U^{*}$-Cauchy filter and so there is a $G \in \mathscr{F}$ such that $G \times G \subset U$. Let $x \in F \cap G$; then $U(x) \cap U^{-1}(x) \in \mathscr{F}$.

Proposition 3.3. Let $(X, \mathcal{U})$ be a totally bounded quasi-uniform space. Then every maximal regular filter is a minimal $U^{*}$-Cauchy filter.

Proof. Let $\mathscr{F}$ be a maximal regular filter. By the preceding lemma, $\mathscr{F}$ is a $U^{*}$-Cauchy filter so that by [4, Proposition 3.30] it suffices to show that $\mathscr{B}=\{U(F): U$ is a symmetric entourage in $U^{*}$ and $\left.F \in \mathscr{F}\right\}$ is a base for $\mathscr{F}_{\text {. Let }} F \in \mathscr{F}$. There is a $U \in \mathscr{U}$ and an $E \in \mathscr{F}$ such that $U^{-1}(E) \subset F$. Evidently, $U \cap U^{-1} \in \mathscr{B}$ and $U \cap U^{-1}(E) \subset F$.

Proposition 3.4. Let $(X, \mathcal{U})$ be a point-symmetric quasi-uniform space. Then, for each $x \in X, \eta^{*}(x)$ is a maximal regular filter.

Proof. Let $x \in X$. Since $(X, \mathscr{U})$ is point symmetric, $\left\{U^{-1}(x): U \in \mathscr{U}\right\}$ is a base for $\eta^{*}(x)$. Let $U \in \mathcal{U}$ and let $V \subset U$ such that $V^{2} \subset U$. Then $V^{-1}\left(V^{-1}(x)\right) \subset U^{-1}(x)$ and so $\eta^{*}(x)$ is a regular filter. The result follows from Lemma 3.1.

THEOREM 3.5. Let $(X, \mathcal{U})$ be a point-symmetric totally bounded $T_{1}$ quasi-uniform space. Then $(X, \mathcal{U})$ has a totally bounded compactification $(X, \breve{U})$ that is a subspace of the bicompletion of $(X, \mathcal{U})$. Moreover, if $\mathcal{U}$ is a uniformity, $(\breve{X}, \widetilde{U})$ is the uniform completion of $(X, \mathcal{U})$.

Proof. Let $\breve{X}$ denote the set of all maximal regular filters on $X$. By Proposition 3.3,

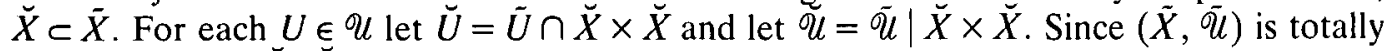
bounded, so is $(\bar{X}, \breve{U})$.

To show that $(\breve{X}, \breve{U})$ is a $T_{1}$ space, let $\mathscr{F}$ and $\mathscr{S}$ be two members of $X$ and suppose that $(\mathscr{F}, \mathscr{G}) \in \cap \breve{\mathscr{U}}$. Since $\mathscr{F}$ and $\mathscr{G}$ are maximal regular filters, there exist $F \in \mathscr{F}$ and $G \in \mathscr{G}$ such that $F \cap G=\varnothing$. As $\mathscr{G}$ is a regular filter, there exist $U \in \mathscr{U}$ and $G_{1} \in \mathscr{G}$ such 
that $U^{-1}\left(G_{1}\right) \subset G$. Since $(\mathscr{F}, \mathscr{G}) \in \breve{\mathscr{U}}$, there exist $F_{2} \in \mathscr{F}$ and $G_{2} \in \mathscr{G}$ such that $F_{2} \times G_{2} \subset U$. Let $x \in F \cap F_{2}$ and $y \in G_{1} \cap G_{2}$. Then $x \in F \cap U^{-1}(y) \subset F \cap U^{-1}\left(G_{1}\right) \subset F \cap G=\varnothing$-a contradiction.

The map $i: X \rightarrow \tilde{X}$ defined by $i(x)=\eta^{*}(x)$ is a quasi-uniform embedding and, by Proposition 3.4, $i(X) \subset \breve{X}$. Furthermore $i(X)$ is a dense subspace of $\left(\tilde{X}, \mathscr{T}\left(\tilde{U}^{*}\right)\right)$ and therefore $i(X)$ is a dense subset of $\left(\check{X}, \mathscr{T}\left(\mathscr{U}^{*}\right)\right)$.

We show that $(\bar{X}, \breve{U})$ is compact. By Proposition 2.2(a), it suffices to show that every regular filter on $\breve{X}$ has a cluster point. Let $\mathcal{M}$ be a regular filter on $\breve{X}$. Since $i(X)$ is a $T\left(U^{-1}\right)$-dense subset of $X,\left\{i^{-1}(M): M \in \mathcal{M}\right\}$ is a base for a filter $\mathscr{F}$ on $X$. It is a routine matter to show that $\mathscr{F}$ is a regular filter. Let $\mathscr{G}$ be a maximal regular filter containing $\mathscr{F}$. We show that $\mathscr{G}$, as a point of $\breve{X}$, is a $\mathscr{T}(\breve{U})$-cluster point of $\mathcal{M}$. Let $U \in \mathscr{U}, V \in \mathscr{U}$ such that $V^{2} \subset U$ and let $M \in M$. Since $i^{-1}(M) \in \mathscr{G}$, by Proposition 3.2 there exists an $x$ in $i^{-1}(M)$ such that $V^{-1}(x) \in \mathscr{G}$. As $V(x) \in \eta^{*}(x)$ and $V^{-1}(x) \times V(x) \subset U, \eta^{*}(x) \in U(\mathscr{G}) \cap M$.

Finally, if $u$ is a uniformity, $(\bar{X}, \tilde{U})$ coincides with the standard completion of a uniform space by means of regular Cauchy filters [1, Page 101].

The following corollary is a curious consequence of the preceding theorem and Proposition 2.1.

Corollary. Let $(X, \mathcal{U})$ be a totally bounded point-symmetric $T_{1}$ space. Then $\boldsymbol{u}$ is a uniformity if and only if every minimal $U^{*}$-Cauchy filter is a maximal regular filter.

In general, a totally bounded quasi-uniform space may have many totally bounded compactifications; indeed, if $\mathscr{P}$ denotes the Pervin quasi-uniformity of a Tychonoff space $X$ and $\mathscr{P}$ denotes the Pervin quasi-uniformity of any Hausdorf compactification $\hat{X}$ of $X$, then $(\hat{X}, \hat{\mathscr{P}})$ is a totally bounded compactification of $(X, \mathscr{P})$. [3, Proposition, Page 203]. The remaining results indicate the well-behaviour of the compactification selected by the construction of Theorem 3.5.

Proposition 3.6. Let $(X, \mathcal{U})$ be a point-symmetric $T_{1}$ quasi-uniform space, let $\mathscr{F}$ be a maximal regular filter on $X$, and let $x$ be a cluster point of $\mathscr{F}$. Then $\mathscr{F}=\eta^{*}(x)$.

Proof. Since $\eta^{*}(x)$ is a regular filter, it suffices to show that $\mathscr{F} \subset \eta^{*}(x)$. Let $\mathscr{B}=\{F \in \mathscr{F}: F=\bar{F}\}$. Then $x \in \bigcap \mathscr{B}$ and $\mathscr{B}$ is a base for $\mathscr{F}$. Let $U \in \mathscr{U}$ and $B \in \mathscr{B}$. Then $U \cap U^{-1}(x) \subset U^{-1}(B)$ and so $U^{-1}(B) \in \eta^{*}(x)$. Thus $\mathscr{F}=\mathscr{F} r \subset \eta^{*}(x)$.

Corollary. If $(X, U)$ is a compact totally bounded $T_{1}$ quasi-uniform space, $X=\check{X}$.

THEOREM 3.7. Let $(X, \mathcal{U})$ be a totally bounded point-symmetric $T_{1}$ quasi-uniform space, let $(Y, \mathscr{V})$ be a totally bounded compact $T_{1}$ quasi-uniform space, and let $f:(X, \mathscr{U}) \rightarrow(Y, \mathscr{V})$ be a quasi-uniformly continuous map. If $f$ maps $X$ onto $Y$, or $\mathscr{V}$ is a uniformity, then $f$ has a quasi-uniformly continuous extension $\breve{f}:(X, \breve{U}) \rightarrow(Y, \mathscr{V})$.

Proof. By [4, Theorem 3.29], there is a $\tilde{U}-\tilde{V}$ quasi-uniformly continuous map $g: X \rightarrow Y$ defined for each minimal $U^{*}$-Cauchy filter $\mathscr{F}$ by $g(\mathscr{F})=\mathscr{T}\left(\tilde{\mathscr{V}}^{*}\right)$-limit fil $\{f(F): F \in \mathscr{F}\}$. Let $\breve{f}=g \mid \check{X}$. If $\mathscr{V}$ is a uniformity, $(Y, \mathscr{V})=(\tilde{Y}, \tilde{\mathscr{V}})$ and we are finished. 
Now suppose that $f$ maps $X$ onto $Y$ and let $\mathscr{F} \in X$. Then, as is easily verified, $\{f(F): F \in \mathscr{F}\}$ is a base for a maximal $\mathscr{V}$-regular filter $\mathscr{H}$; we show that $\mathscr{H}$, considered as a point of $\breve{Y}$, is $\breve{f}(\mathscr{F})$. Since $\mathscr{T}\left(\tilde{\mathscr{V}}^{*}\right)$ is a Hasudorff topology, it suffices to show that $\mathscr{H}$ is a $\mathscr{T}\left(\tilde{V}^{*}\right)$-cluster point of $\mathscr{H}$. To this end, let $F \in \mathscr{F}$, let $V \in \mathscr{V}$, and let $W \in V$ so that $W^{2} \subset V$. By Proposition 3.2, there is a $y \in f(F)$ so that $W(y) \cap W^{-1}(y) \in \mathscr{H}$. Since $W^{-1}(y) \in \eta^{*}(y)$ and $W(y) \times W^{-1}(y) \subset V^{-1}, \quad \eta^{*}(y) \in \bar{V}^{-1} \times(\mathscr{H}) \cap f(F)$. Similarly, we see that $\eta^{*}(y) \in \tilde{V}(\mathscr{H}) \cap f(F)$, and so $\mathscr{H}$ is a $\mathscr{T}\left(\tilde{V}^{*}\right)$-cluster point of $\mathscr{H}$. By the previous corollary, $Y=\breve{Y}$, and so $\breve{f}$ maps into $Y$ as required.

Any continuous map between two topological spaces is a quasi-uniformly continuous map between the two corresponding Pervin quasi-uniform spaces. The extension property established in the previous theorem suggests, therefore, that the compactification $(X, \breve{P})$ might be of particular interest. In considering this compactification, we use the following standard notation: For any subset $A$ of a set $X, A^{*}$ denotes $\{\mathscr{F}: \mathscr{F}$ is a maximal closed filter on $X$ and $A \in \mathscr{F}\}$ and $S(A)=T(A, X-A)$. A subbase for the Pervin quasiuniformity of a topological space $(X, \mathscr{T})$ is $\{S(A): A \in \mathscr{T}\}$ and a base for the topology of the Wallman compactification of a $T_{1}$-space $(X, \mathscr{T})$ is $\left\{G^{*}: G \in \mathscr{T}\right\}$.

THEOREM 3.8. Let $X$ be a $T_{1}$-space and let $\mathscr{P}$ be the Pervin quasi-uniformity for $X$. Then $(\breve{X}, \mathscr{T}(\breve{P}))$ is the Wallman compactification of $X$.

Proof. We take $\hat{X}$ to be the collection of all filters on $X$ that are maximal with respect to the property of having a closed base; since a filter has a closed base if and only if it is $\mathscr{P}$-regular, $\breve{X}=\hat{X}$.

Let $\mathscr{P}$ be the Pervin quasi-uniformity for $\hat{X}$. To see that $\breve{\mathscr{P}} \subset \hat{\mathscr{P}}$, let $E$ be a closed subset of $X$ and let $U=S(X-E)$. We show that $\breve{U}=S\left(\breve{X}-E^{*}\right)$. Let $(\mathscr{F}, \mathscr{G}) \in \breve{U}$. If $\mathscr{F} \in E^{*}$, it is obvious that $(\mathscr{F}, \mathscr{G}) \in S\left(X-E^{*}\right)$. If $\mathscr{F} \in E^{*}$, there exists an $F \in \mathscr{F}$ and a $G \in \mathscr{G}$ such that $F \times G \subset U$ and $F \cap E=\varnothing$. It follows that $G \subset X-E$ so that $\mathscr{G} \notin E^{*}$; hence $(\mathscr{F}, \mathscr{G}) \in S\left(\tilde{X}-E^{*}\right)$. Now suppose that $(\mathscr{F}, \mathscr{G}) \in S\left(\breve{X}-E^{*}\right)$. If $\mathscr{F} \in E^{*}$, then $E \in \mathscr{F}$ and $X \in \mathscr{G}$ so that $(\mathscr{F}, \mathscr{G}) \in \breve{U}$. If $\mathscr{F} \notin E^{*}$, then $X-E \in \mathscr{F} \cap \mathscr{G}$ so that $(\mathscr{F}, \mathscr{G}) \in \breve{U}$. Thus $\mathscr{T}(\breve{P})$ is coarser than the topology of the Wallman compactification of $X$.

To see that $\mathscr{T}(\hat{\mathscr{P}}) \subset \mathscr{T}(\mathscr{P})$, let $G$ be a $\mathscr{T}(\hat{\mathscr{P}})$-open set, let $\mathscr{F} \in G$ and let $E=\breve{X}-G$. Then $E=\bigcap\left\{E_{\alpha}^{*}: \alpha \in A\right\}$ where, for each $\alpha \in A, E_{\alpha}$ is a closed subset of $X$. There exists $\alpha \in A$ so that $\mathscr{F} \notin E_{\alpha}^{*}$. Since $S\left(\breve{X}-E_{\alpha}^{*}\right)$ is an entourage of $\hat{\mathscr{P}}, V=S\left(\breve{V}-E_{\alpha}^{*}\right) \cap X \times X$ is an entourage of the Pervin quasi-uniformity on $X$. It suffices to show that $\breve{V}(\mathscr{F}) \subset G$. Suppose that $\mathscr{H} \in \breve{V}(\mathscr{F}) \cap E$. There exist $F \in \mathscr{F}$ and $H \in \mathscr{H}$ so that $F \times H \subset V=X \times X-$ $\left(X-E_{\alpha} \times E_{\alpha}\right)$. Since $\mathscr{F} \notin E_{\alpha}^{*}$, we assume, without loss of generality, that $F \subset X-E_{\alpha}$. Thus $H \cap E_{\alpha}=\varnothing$; and, since $\mathscr{H} \in E \subset E_{\alpha}^{*}$, we have a contradiction.

Our final result establishes a sufficient condition in order that $(\breve{X}, \breve{U})$ be a Hausdorff compactification. This condition is easily seen to be satisfied by a $T_{1}$ totally bounded quasi-uniform space that is either normal and equinormal or a uniform space.

We say that a relation $V$ on a set $X$ separates subsets $A$ and $B$ of $X$ provided that $V(A) \cap V(B)=\varnothing$. A quasi-uniform space $(X, \mathscr{U})$ satisfies property ${ }^{*}$ provided that any 
two subsets of $X$ that are separated by a member of $\mathcal{U}^{-1}$ are also separated by a member of $u$.

Proposition 3.9. Let $(X, \mathcal{U})$ be a point-symmetric totally bounded $T_{1}$ quasi-uniform space satisfying property *. Then $(\breve{X}, \breve{\mathscr{U}})$ is a Hausdorff compactification of $(X, \mathcal{U})$.

Proof. Let $\mathscr{F}$ and $\mathscr{G}$ be two members of $\breve{X}$. There is an $A \in \mathscr{F}$, and $B \in \mathscr{G}$, and a $U \in \mathcal{U}$ so that $U^{-1}(A) \cap U^{-1}(B)=\varnothing$. By hypothesis there is a $V \in \mathcal{U}$ with $V(A) \cap V(B)=\varnothing$. Let $W \in \mathscr{U}$ with $W^{2} \subset V$. We assert that $\tilde{W}(F) \cap \tilde{W}(G)=\varnothing$. Suppose that $\mathscr{H} \in \tilde{W}(\mathscr{F}) \cap$ $\bar{W}(\mathscr{G})$. Then there is an $F \in \mathscr{F}$, a $G \in \mathscr{G}$, and an $H \in \mathscr{H}$ such that $F \times H \subset W, G \times H \subset W$, and $H \times H \subset W$. Thus $F \times G \subset W \circ W \circ W^{-1} \subset V \circ V^{-1}$. Since there exists $(p, q) \in(F \times$ $G) \cap(A \times B)$, there is an $r \in X$ such that $(p, r) \in V$ and $(r, q) \in V^{-1}$; hence $r \in V(p) \cap$ $V(q) \subset V(A) \cap V(B)$-a contradiction.

According to Theorem 3.47 of reference [4], a totally bounded Tychonoff space $(X, \mathcal{U})$ has a Hausdorff compactification if and only if $U$ contains a uniformity compatible with $\mathscr{T}(\mathscr{U})$. Thus any point-symmetric totally bounded Tychonoff quasi-uniformity $\mathscr{U}$ satisfying property * contains a uniformity compatible with $\mathscr{T}(U)$. If $X$ is a Tychonoff space that is not normal, then $(X, \mathscr{P})$ has a Hausdorff compactification, but $(\breve{X}, \breve{P})$ is the Wallman compactification, which fails to be a Hausdorff space. Thus a quasi-uniformity $\mathcal{U}$ may contain a uniformity compatible with $\mathscr{T}(U)$ and still fail to satisfy property ${ }^{*}$. The problem of determining necessary and sufficient conditions in order that $(\breve{X}, \breve{u})$ be a Hausdorff compactification is still open; indeed, even property ${ }^{*}$ has not yet been ruled out as such a condition.

\section{REFERENCES}

1. E. M. Alfsen and J. E. Fenstad, A note on completions and compactifications, Math. Scand. 8 (1960), 97-104.

2. Á. Császár, Complete extensions of quasi-uniform spaces, General topology and its relations to modern analysis and algebra Proc. Sympos., Prague, 1981 (Heldermann Verlag, 1982), 104-113. 200-204.

3. P. Fletcher, On completeness of quasi-uniform spaces, Arch. Math. (Basel) 22 (1971),

4. P. Fletcher and W. F. Lindgren, Quasi-uniform spaces, Lecture Notes in Pure and Applied Mathematics 77 (Marcel Dekker, 1982).

5. O. Njåstad, On Wallman-type compactifications, Math. Z. 91 (1966), 267-276.

Department of Mathematics

Virginia Polytechnic Institute and State University

BLACKSBURG, VIRGINIA 24061-4097

U.S.A.

Department of Mathematics

Slippery Rock State University

Slippery Rock, Pennsylvania 16057

U.S.A. 